\title{
Hyponatremia may lead to hepatic encephalopathy
}

A new study reveals that hyponatremia (serum sodium levels $<130 \mathrm{mmol} / \mathrm{l}$ ) is a risk factor for the development of overt hepatic encephalopathy (OHE) in patients with cirrhosis, and that its treatment may be a novel therapeutic approach to prevent $\mathrm{OHE}$.

Hyponatremia is suggested to induce changes in brain osmolytes that are similar to those that occur during hyperammonemia in patients with cirrhosis. "These changes may participate in the development of OHE", explains Monica Guevara from the Hospital Clinic, Barcelona, Spain. Their study specifically assesses the relationship between serum sodium levels and the development of $\mathrm{OHE}$ in patients with cirrhosis.

The team evaluated episodes of $\mathrm{OHE}$ over a 1-year period in 61 patients with cirrhosis. Clinical and biochemical data were gathered at baseline, 3-month intervals, and when OHE developed. In addition, 25 patients underwent ${ }^{1} \mathrm{H}$-magnetic resonance spectroscopy at baseline to determine concentrations of organic brain osmolytes and their relationships to serum sodium levels. A time-dependent statistical analysis was performed. "We thought this approach was the best as OHE is a recurrent event and the variables related to its development may change over time", explains Guevara. Results show that hyponatremia is an independent predictor of risk of $\mathrm{OHE}$ and is associated with low levels of organic brain osmolytes, particularly myo-inositol. The probability of developing OHE was higher in patients with low myo-inositol levels than high levels.

"Studies should be designed to evaluate [whether] treatment of hyponatremia with vasopressin-receptor antagonists can reduce the incidence of $\mathrm{OHE}$,' Guevara concludes.

\section{Rachel Jones}

Original article Guevara, M. Hyponatremia is a risk factor of hepatic encephalopathy in patients with cirrhosis: a prospective study with time-dependent analysis. Am. J. Gastroenterol. 104, 1382-1389 (2009). 